\title{
Análisis de crecimiento durante la fase vegetativa de cinco fenotipos de copoazú Theobroma grandiflorum (Willd ex Spreng) Schum. en la amazonia occidental colombiana
}

\author{
Growth analysis during the vegetative phase of five \\ phenotypes of copoazú Theobroma grandiflorum \\ (Willd ex Spreng) Schum. in the Colombian western amazonia
}

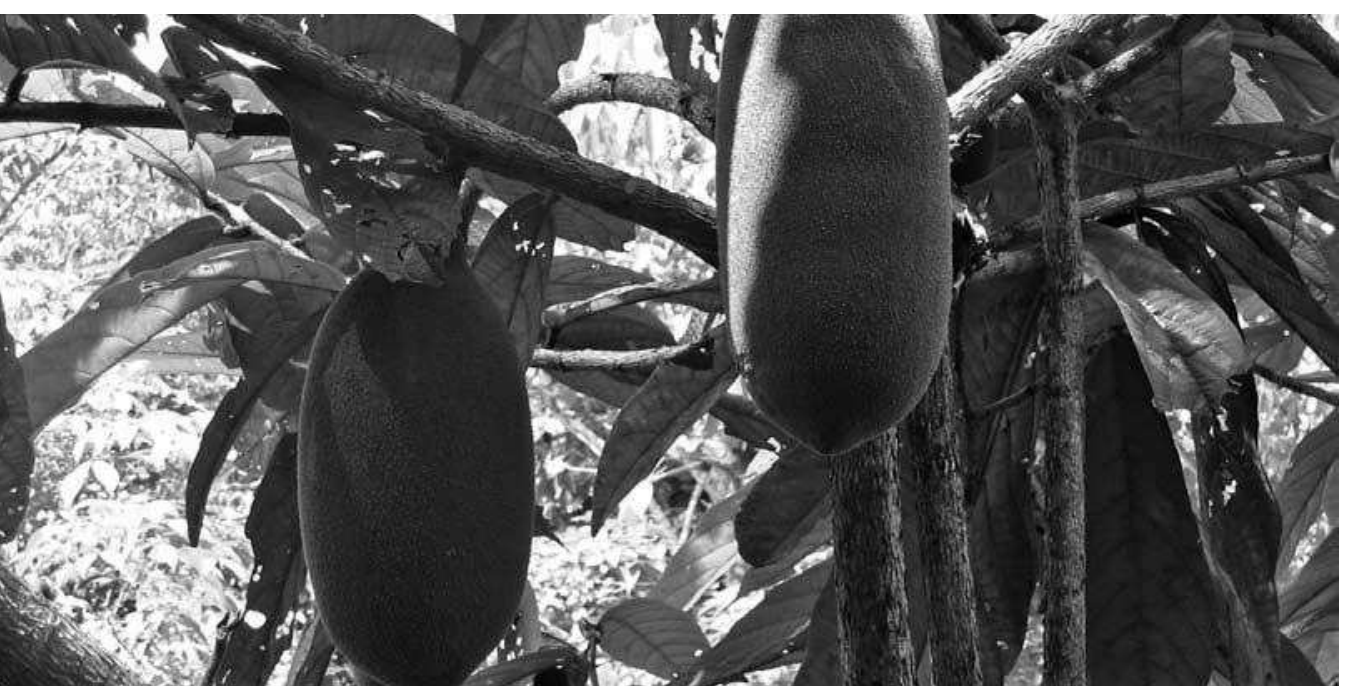

DIANA CAROLINA GUERRERO'

JAIME ALBERTO BARRERA ${ }^{2}$

MARÍA SOLEDAD HERNÁNDEZ $Z^{3}$

GUILLERMO VARGAS ${ }^{4}$

Árbol de copoazú en fructificación.

Foto: M. S. Henández

\section{RESUMEN}

El trabajo se desarrolló en cuatro fincas del departamento de Caquetá, con el objetivo de analizar el comportamiento fisiológico durante las etapas tempranas de crecimiento de cinco fenotipos de copoazú colección Sinchi bajo diferentes coberturas de dosel de árboles. Para tal efecto se utilizó un diseño experimental de bloques, en el cual se distribuyeron al azar los cinco fenotipos de copoazú. Durante el periodo de evaluación se determinaron variables de altura, área foliar, masa seca y tasas de crecimiento. Las variables directas mostraron un incremento constante especialmente en tamaño y en ganancia de materia seca, además se presentó un comportamiento estable en la duración del área foliar; por el contrario, las tasas de crecimiento como la tasa de asimilación neta, tasa de crecimiento relativo, índice del área foliar y la relación del área foliar, presentaron valores fluctuantes en los cinco ecotipos a lo largo del periodo de evaluación. Este resultado indica un comportamiento diferencial en

Investigadora contratista, Instituto Amazónico de Investigaciones Científicas SINCHI, sede Florencia. diacarog@yahoo.es Investigador, Instituto Amazónico de Investigaciones Científicas SINCHI, sede Bogotá. jbarrera@sinchi.irg.co Investigadora, Instituto Amazónico de Investigaciones Científicas SINCHI, sede Bogotá. fhernandez@sinchi.org.co Investigador, Instituto Amazónico de Investigaciones Científicas SINCHI, sede Guaviare.gvargas@sinchi.org.co 
las primeras fases de crecimiento del copoazú, lo que hace presumir que hay una interacción genotipo ambiente reflejada en los diferentes comportamientos de los determinantes fisiológicos de la planta evidenciando cierta plasticidad fenotípica de acuerdo con cada una de las coberturas, encontrándose mejor comportamiento bajo un $15 \%$ de sombreamiento. Los ecotipos 4 y 11 se diferenciaron del resto de ecotipos por presentar mejor adaptabilidad en todas las localidades independientemente del porcentaje de cobertura. Estudios previos mostraron que estos ecotipos según sus características morfoagronómicas presentan una alta productividad y se encuentran en grupos caracterizados por diferentes niveles de tolerancia a los ataques de la escoba de bruja (Crinipelis perniciosa).

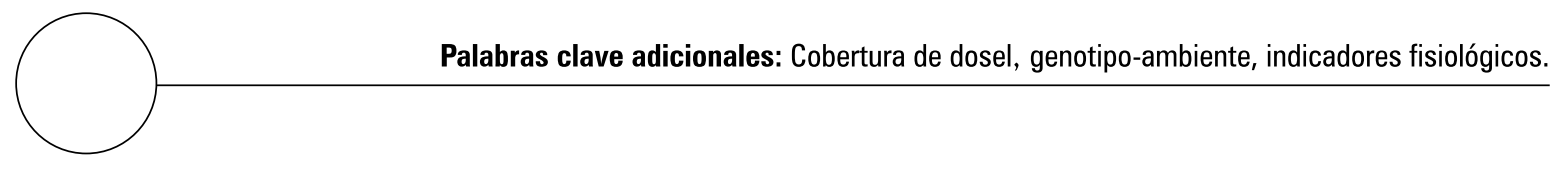

\section{ABSTRACT}

The research was undertaken in four farms of Caquetá department in order to analyze physiologic responses of five copoazu phenotypes, Sinchi collection at the early growth stages under different canopy coverings. For such an effect, an experimental design of blocks was used with five copoazu phenotypes distributed at random. During the period of evaluation, variables of height, leaf area, dry mass, and rates of growth were determined. The direct variables had a constant increment, especially in size and gain of dry matter, and also presented a stable behavior in the duration of leaf area. On the contrary, the rates of growth, assimilation rate, relative growth rate, leaf area index, and leaf area ratio presented oscillating values in five ecotypes during the period of evaluation. These results indicated a differential behavior of copoazu in the first phases of growth that indicated an interaction of a genotype with an environment reflected in the different behaviors of physiologic determinants of the plant and evidenced certain plasticity in phenotypes, according to each of the covering, being the best one under $15 \%$ coverage. The ecotypes 4 and 11 were differed from the other ecotypes presenting better adaptability in all sites independently of the covering percentage. Previous studies showed that, according to their morpho-agronomic characteristics, these ecotypes presented a high productivity and are included into the group characterized by different levels of tolerance to the attacks of witch broom (Crinipelis perniciosa).

Additional key words: Canopy cover, genotype-environment, physiological indicators.
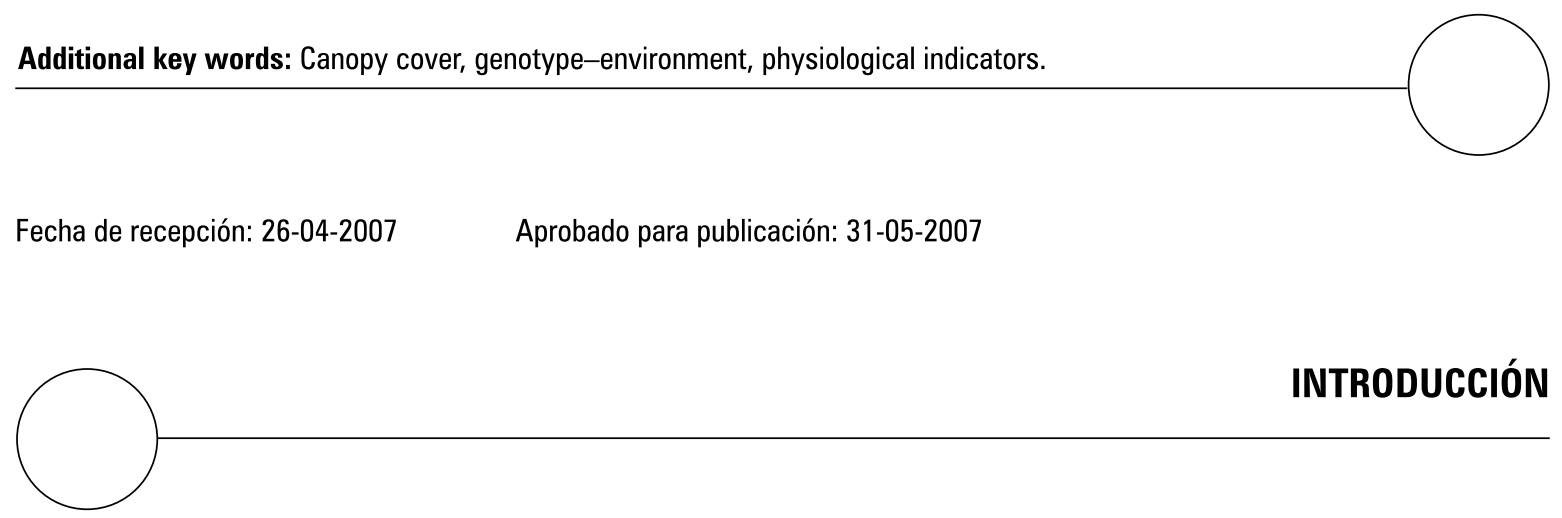

Por la gran variedad de especies comestibles silvestres que ofrece la cuenca amazónica, sus formas, colores y características organolépticas las hace interesante para el consumo humano, siendo ésta una gran alternativa para el mejoramien- to de la canasta familiar. El copoazú Theobroma grandiflorum (Willd ex Spreng) Schum., es una especie frutícola tropical, cuya importancia económica radica en su fruto que puede ser aprovechado en su totalidad (Rojas et al., 1996). 
Estas y muchas otras especies son consideradas como promisorias en la agroindustria por sus muchas formas de utilización. Por ello, mediante un banco de germoplasma se pretende conservar, caracterizar y evaluar las especies de importancia económica en la región. Y a partir de ello, identificar cuales de éstas pueden ser utilizadas en el mejoramiento de la producción agraria, ofreciendo de esta manera especies de frutales que ayuden a diversificar la producción y crear un entorno más armónico y estable.

En este estudio, se analizó la fase temprana de crecimiento vegetativo de cinco fenotipos de copoazú; los cuales fueron seleccionados del banco de germoplasma que custodia el Instituto SINCHI en el departamento de Guaviare, por registrar individuos con características deseables que se puedan identificar desde los estadios tempranos de su crecimiento. El objetivo fue observar cuales de estos ecotipos tienen mejor comportamiento y de esta forma seleccionar aquellos que presentan mejores características, para así implementar y enriquecer los sistemas productivos agrarios.

\section{MATERIALES Y METODOLOGÍA}

\section{Localización}

Este trabajo se desarrolló en cuatro localidades del departamento de Caquetá; la primera de ellas al norte de Florencia en jurisdicción del municipio de la Montañita, denominada finca Bella Vista, ubicada a $1^{\circ} 28^{\prime} 47,4^{\prime \prime}$ N y $75^{\circ} 24^{\prime} 41,6^{\prime \prime}$ $W$ en la vía que conduce a este municipio desde Florencia. La segunda localidad se denomina finca Villa Nueva, situada a $1^{\circ} 24^{\prime} 37,7^{\prime \prime} \mathrm{N}$ y $75^{\circ}$ $46^{\prime} 07,2^{\prime \prime} \mathrm{W}$ en el kilómetro 5 de la vía Morelia-Valparaíso al sur de Florencia. La tercera localidad se denomina finca Paraíso Amazónico, localizada a $1^{\circ} 43^{\prime} 42,6^{\prime \prime} \mathrm{N}$ y $75^{\circ} 39^{\prime}$ 05,6”'W en la vía que conduce de Florencia al departamento de Huila hacia el oriente; y por último la finca San Francisco 2, localidad ubicada a $1^{\circ} 28^{\prime} 39,5^{\prime \prime}$
N y $75^{\circ} 34^{\prime} 28,6^{\prime \prime}$ W en el corregimiento de San Juan del Barro, vereda cercana al casco urbano de Florencia.

Las parcelas se ubicaron en paisajes de piedemonte y lomerío, bajo un ambiente de bosque húmedo tropical característico de la región, donde su temperatura oscila entre los 18 a $20^{\circ} \mathrm{C}$ la mínima y 30 a $35^{\circ} \mathrm{C}$ la máxima; con una precipitación anual entre los 3.000 y $4.000 \mathrm{~mm}$, con distribución monomodal y una humedad relativa de 75 a $85 \%$.

\section{Material vegetal}

El material vegetal se seleccionó del banco de germoplasma de la colección Sinchi, ubicado en la granja experimental El Trueno, situada en el departamento del Guaviare, en donde se tienen 16 accesiones de $T$. grandiflorum debidamente identificadas y caracterizadas morfoagronómicamente y molecularmente por la técnica de AFL'Ps. Se seleccionaron cinco ecotipos cuyas características se describen en la tabla 1.

\section{Establecimiento}

El enriquecimiento agroforestal se llevó a cabo en una hectárea de bosque intervenido en cada una de las localidades seleccionadas. Las plantas fueron distribuidas experimentalmente en tres bloques divididos entre sí en cinco subparcelas, en cada una de las cuales fueron distribuidos de forma aleatoria cuatro árboles de cada ecotipo de copoazú.

La distribución de las plantas en la parcela fue: los maderables de nogal se ubicaron cada $4 \mathrm{~m}$ entre plantas y 16 m entre surcos dando un número de individuos de 150; el copoazú fue distribuido a 4 m entre plantas y 16 m entre surcos con un total de 150 plántulas, de los cuales 60 individuos se seleccionaron como materiales de evaluación. De esta forma se evaluaban cuatro árboles de cada ecotipo por tres réplicas para un total de 12 individuos de cada material. 
Tabla 1. Descripción morfoagronómica de de los ecotipos de copoazú objeto del presente estudio.

\begin{tabular}{|c|c|c|c|}
\hline Ecotipos & Botánica & Morfología & $\begin{array}{l}\text { Grupo susceptible a } \\
\text { enfermedades }\end{array}$ \\
\hline Código 3-002 & $\begin{array}{l}\text { Tallo densamente ferrugineo-fasciculado. Hoja } \\
\text { simple, alterna, obovada-oblonga. La lámina } \\
\text { foliar varia entre } 19,5-32 \text { × 4,8-13 cm. Con } \\
\text { nervaduras glabras con } 10-11 \text { pares de nervios } \\
\text { secundarios, pecíolo de } 1 \text { a } 2 \mathrm{~cm} \text { de longitud. }\end{array}$ & $\begin{array}{l}\text { Fruto de forma oblonga con un } \\
\text { peso promedio de } 865,5 \mathrm{~g} \text {. Peso } \\
\text { de la pulpa de } 262,7 \mathrm{~g} \text {, semilla de } \\
382,5 \mathrm{~g} \text {; una producción media } \\
\text { anual por árbol de } 26 \mathrm{~kg} \text {. }\end{array}$ & $\begin{array}{l}\text { Grupo } 2,3,4 \text { alta, } \\
\text { mediana y poco } \\
\text { tolerables. }\end{array}$ \\
\hline Código 3-004 & $\begin{array}{l}\text { Tallo densamente ferrugineo-fasciculado. } \\
\text { Hojas simples obovadas oblongas. La lámi- } \\
\text { na foliar varia entre } 21,5-36 \times 7,3-9 \mathrm{~cm} \text {. Las } \\
\text { nervaduras glabras con } 9-11 \text { pares de nervios } \\
\text { secundarios. }\end{array}$ & $\begin{array}{l}\text { Frutos de forma oblonga con un } \\
\text { peso promedio de } 1.486,6 \mathrm{~g} \text {. Peso } \\
\text { de pulpa de } 439 \mathrm{~g} \text { y de las semi- } \\
\text { llas de } 672 \mathrm{~g} \text {; una producción me- } \\
\text { dia anual por árbol de } 44,6 \mathrm{~kg} \text {. }\end{array}$ & $\begin{array}{l}\text { Grupo } 2 \text { altamente } \\
\text { tolerable. }\end{array}$ \\
\hline Código 3-005 & $\begin{array}{l}\text { Tallo densamente ferrugineo-fasciculado a gla- } \\
\text { brescente. Hojas simples alternas obovadas } \\
\text { oblongas. La lámina foliar varia entre } 23-31 x \\
6,5-10,5 \mathrm{~cm} \text {. Las nervaduras glabras con } 8-11 \\
\text { pares de nervios secundarios, pecíolo de } 1 \mathrm{~cm} \\
\text { de longitud. }\end{array}$ & $\begin{array}{l}\text { Fruto de forma oblonga con un } \\
\text { peso promedio de } 1.250 \mathrm{~g} \text {, las } \\
\text { semillas pesan } 543 \mathrm{~g} \text { y la pulpa } \\
340,7 \mathrm{~g} \text {. Producción media anual } \\
\text { por árbol de } 37,5 \mathrm{~kg} \text {. }\end{array}$ & $\begin{array}{l}\text { Grupo } 1 \text { plus o posi- } \\
\text { blemente resistentes. }\end{array}$ \\
\hline Código 3-011 & $\begin{array}{l}\text { Tallo densamente fasciculado a glabrescente } \\
\text { estriado. Hojas simple alterna, el tamaño de la } \\
\text { lámina foliar es de } 14-33 \text { × } 5-11,5 \mathrm{~cm} \text {. Las ner- } \\
\text { vaduras glabras con } 8-11 \text { pares de nervios se- } \\
\text { cundarios, pecíolo de } 0,8 \text { a } 1,5 \mathrm{~cm} \text { de longitud. }\end{array}$ & $\begin{array}{l}\text { Fruto de forma oblonga con un } \\
\text { peso promedio de } 1.800 \mathrm{~g} \text {, peso } \\
\text { de la pulpa } 495 \mathrm{~g} \text { y de la semilla } \\
792 \mathrm{~g} \text {. Una producción media } \\
\text { anual por árbol de } 54 \mathrm{~kg} \text {. }\end{array}$ & $\begin{array}{l}\text { Grupo } 4 \text { poco tole- } \\
\text { rantes. }\end{array}$ \\
\hline Código 3-015 & & $\begin{array}{l}\text { Frutos de forma oblonga con un } \\
\text { peso promedio de } 1.290 \mathrm{~g} \text {, peso } \\
\text { de la pulpa } 460 \mathrm{~g} \text { y un número de } \\
\text { semillas por fruto de } 32 \text {. }\end{array}$ & $\begin{array}{l}\text { Grupo } 1,2 \text { y } 4 \text { árboles } \\
\text { plus altamente tole- } \\
\text { rantes a poco. }\end{array}$ \\
\hline
\end{tabular}

Al momento del establecimiento de los árboles se adicionó una dosis de abono orgánico tipo gallinaza en mezcla con un material de encalado tipo cal dolomita, con el propósito de atenuar las restricciones que se presentan en los análisis de caracterización de suelos realizados a cada localidad.

\section{Coberturas arbóreas}

Se entiende por cobertura el cálculo en metros cuadrados y porcentaje del área que proyecta so- bre el suelo la copa de cada individuo arbóreo de los estratos altos (Rangel et al., 1997). En cada una de las parcelas se delimitó un área de $10 \mathrm{x}$ $100 \mathrm{~m}$, en donde se midió a los árboles con DAP (diámetro a la altura del pecho) mayor de $10 \mathrm{~cm}$, altura total y comercial, además el diámetro mayor y menor de la copa; de donde posteriormente se obtendrían los datos de sombra proyectada sobre el lote, después de realizar la sumatoria de la totalidad de individuos de cada superficie de acuerdo con la siguiente expresión matemática: 
Cobertura de copa de cada individuo:

$\mathrm{C}=1 / 2(\mathrm{D} 1 \times \mathrm{D} 2)$
$\mathrm{D} 1=$ Diámetro mayor de la copa
$\mathrm{D} 2=$ Diámetro menor de la copa

Fase de investigación para el análisis de crecimiento

Para determinar el área foliar y el peso seco y dado el valor comercial y científico de los materiales en estudio, además del tamaño reducido de cada parcela se aplicaron sendos modelos matemáticos para el calculo de estas variables. Mediante la toma de medidas directas de crecimiento en el campo como altura, diámetro basal a 0 y $10 \mathrm{~cm}$, radios a y b de las hojas y número de hojas, se puede estimar el valor de peso seco y la magnitud del área foliar de cada árbol en un instante determinado. Dichos modelos se presentan en las siguientes ecuaciones:

$\begin{array}{ll}\text { Ecuación peso seco } & \text { Ecuación área foliar } \\ Y=b A+c C+d B C & Y=(b A+C A B)^{*} N \\ Y=\text { peso seco } & Y=\text { área foliar } \\ A=\text { altura total } & A=\text { radio a de la hoja } \\ B=\text { diámetro basal a } 0 \mathrm{~cm} & B=\text { radio } b \text { de la hoja } \\ C=\text { diámetro basal a } 10 \mathrm{~cm} & b y c=\text { constantes } \\ b, c y d=\text { constantes } & N=\text { número de hojas }\end{array}$

\section{MÉTODO FUNCIONAL DEL ANÁLISIS}

Se marcaron en campo 60 individuos que corresponde a los cinco ecotipos a evaluar. En cada uno de ellos se tomaron medidas de altura de la planta, diámetro basal del tallo a $0 \mathrm{~cm}$ y a $10 \mathrm{~cm}$, radio a y b de las hojas, numero de hojas y el área del dosel y aplicar así las ecuaciones descritas. Para el análisis de crecimiento en etapas tempranas se siguió el método funcional que comprende medidas a intervalos de tiempo mas frecuentes (cada 30 días) (Hunt, 1978; Hunt et al., 1984). El análisis se complementó mediante el cálculo de indicadores que describen el crecimiento de la planta siendo los mas destacados: tasa de asimilación neta (TAN), tasa de crecimiento relativo (TCR), índice del área foliar (IAF), relación del área foliar (RAF) y duración del área foliar (DAF). Este método se caracteriza por el uso que hace de la regresión para predecir con datos directos respuestas biológicas futuras (Hunt, 1978; Hunt et al., 1984).

\section{Diseño experimental}

Se utilizó un modelo de bloques completos al azar, en el que se distribuyeron las 60 plántulas pertenecientes a los cinco ecotipos respectivamente. Con este diseño se busca mantener la variabilidad entre unidades experimentales dentro de un bloque, y maximizar las diferencias entre los mismos (Little y Hills, 1976).

Para el logro de los objetivos referentes al análisis de crecimiento, y como se trataba de evaluar una fase temprana de crecimiento se realizó la toma de datos cada 30 días durante un periodo de seis meses.

Con los datos obtenidos se practicó un análisis de varianza para determinar si existen diferencias significativas entre las variables evaluadas. Consecuentemente se practicó un análisis de regresión a aquellos ecotipos con mejor respuesta acorde a los resultados del análisis de varianza.

\section{RESULTADOS Y DISCUSIÓN}

\section{Coberturas}

En general, los árboles y las demás plantas asociadas que forman las coberturas naturales, influyen directamente en el crecimiento y desarrollo de los sistemas agrícolas allí implementados, pues sus doseles afectan la captación directa de 
radiación solar, precipitación y movimiento del aire a la vez que sus sistemas radiculares ocupan grandes volúmenes de suelo; la absorción de agua, la redistribución de nutrientes y las posibles asociaciones bacterianas/fungales, alterando de esta manera el ambiente de crecimiento (Donoso, 1981).

Aunque no todo se da de una forma negativa, ya que estos lugares ayudan a moderar los cambios de temperatura, en comparación con áreas abiertas, también reducen el promedio de evaporación encontrándose mayor humedad relativa y el aporte de algunos nutrientes a través de la hojarasca. Por otro lado, existen componentes biológicos asociados como los insectos y plantas repelentes que ayudan a dar un control integrado (tabla 2).

\section{Análisis de suelo}

En general las cuatro localidades se caracterizaron por suelos de textura areno arcillosa y franco arcillosa, con un pH extremadamente ácido típico de los suelos amazónicos y por consiguiente un alto porcentaje en saturación de aluminio; mientras que la concentración de materia orgánica, saturación de bases y el fósforo intercambiable se encuentran en los niveles más bajos para la mayoría de los cultivos.

\section{Análisis de parámetros y medidas derivadas de crecimiento}

El comportamiento promedio de los últimos 10 años (según IDEAM) de los valores medios de precipitación, humedad relativa, temperatura y brillo solar, se presenta en la figura 1 . Se observa el comportamiento típico de esta región con régimen de lluvias bimodal de alta temperatura promedio, precipitación y humedad relativa que afectan los determinantes fisiológicos de las plantas en comunidad.

\section{DETERMINANTES FISIOLÓGICOS}

Para el análisis de cada uno de los parámetros se tuvo en cuenta los cuadrados medios del análisis de varianza. En general se encontraron diferencias estadísticas al 5\% y al $1 \%$ para efectos principales y algunas de las interacciones de segundo grado. Las respuestas a los tratamientos se analizan mediante figuras para cada localidad.

\section{Altura}

El crecimiento de una planta es un aumento irreversible del tamaño, el cual va acompañado

Tabla 2. Cobertura arbórea determinada en cada una de las cuatro localidades evaluadas.

\begin{tabular}{|l|c|l|}
\hline \multicolumn{1}{|c|}{ Finca } & Porcentaje de cobertura & \multicolumn{1}{c|}{ Descripción lote } \\
\hline A. Bella Vista (Montañita) & $9,4 \%$ & $\begin{array}{l}\text { Hace parte de un barbecho en donde la mayor parte } \\
\text { de vegetación allí existente corresponde a Braquiaria. }\end{array}$ \\
\hline B. Villa Nueva (Morelia) & $15,8 \%$ & $\begin{array}{l}\text { Pertenece a un rastrojo joven allí podemos encontrar } \\
\text { uno que otro árbol de fuste grueso. }\end{array}$ \\
\hline C. Paraíso Amazónico (Florencia) & $38,4 \%$ & $\begin{array}{l}\text { Hace parte de un rastrojo maduro donde la mayoría } \\
\text { de sus árboles no sobrepasan los 10 cm de DAP }\end{array}$ \\
\hline D. San Francisco (Florencia) & $47,8 \%$ & $\begin{array}{l}\text { Allí se puede encontrar algunos árboles de fuste } \\
\text { grueso clasificándose como bosque secundario. }\end{array}$ \\
\hline
\end{tabular}


de un incremento en el peso seco y área foliar. Al observar las tendencias de altura (figura 2), los cinco ecotipos de copoazú, presentaron un comportamiento diferente en crecimiento, a la vez que mostraron cambios significativos entre localidades debido probablemente a los diferentes niveles de cobertura. La diferencia de altura puede estar asociada, en cada uno de los ecotipos, a que la planta en los lugares en donde hay menor penetración de luz, gastan mayor energía en crecimiento de tallos y expansión del área fotosintética que ayude a ser más eficiente su condición para la captación de radiación (Rojas, 1993).

Por otra parte, al comparar el crecimiento entre ecotipos, podemos observar que la mayor altura fue alcanzada por los ecotipos 3-004 y 3-011; indistintamente de la localidad, siendo el ecotipo 3-011 el de mejor respuesta. Resulta importante resaltar el caso del ecotipo 5 en la localidad D bajo un $47,8 \%$ de cobertura que presenta un comportamiento similar a los ecotipos 4 y 11, dejando entrever una mejor adaptación a condiciones de baja luminosidad de este material, que los ecotipos 2 y 15 los cuales manifestaron en las cuatro localidades los valores más bajos.

\section{Acumulación de peso seco}

La ganancia de materia seca de cada uno de los ecotipos tuvo un comportamiento diferencial acorde con las características propias del ensayo. La producción de peso seco para cada uno de los ecotipos (figura 3) presenta un incremento permanente durante los 167 dds. Siendo mayor la ganancia de materia seca en las localidades A y B con coberturas de 9,4\% y 15,8\%. Nuevamente, se aprecia que los ecotipos $4 \mathrm{y}$ 11 presentan las mejores respuestas indistintamente de la localidad, lo cual es un reflejo claro de la interacción fisiológica genotipo-ambiente, expresada en una mejor eficiencia fotosintética. Muchos estudios alusivos al sombreamiento muestra la importancia de la radiación en la producción de masa seca (Early, 1966; Magalhaes y Motojo, 1971). De acuerdo con Gómez et al. (1999), la producción de materia seca en las plantas es el resultado neto del balance entre los procesos metabólicos básicos. La materia seca es producida en el proceso fotosintético, los productos se acumulan y son distribuidos a los diferentes órganos de la planta. En este caso la elongación del tallo y la producción de hojas constituyeron la mayor expresión de crecimien-
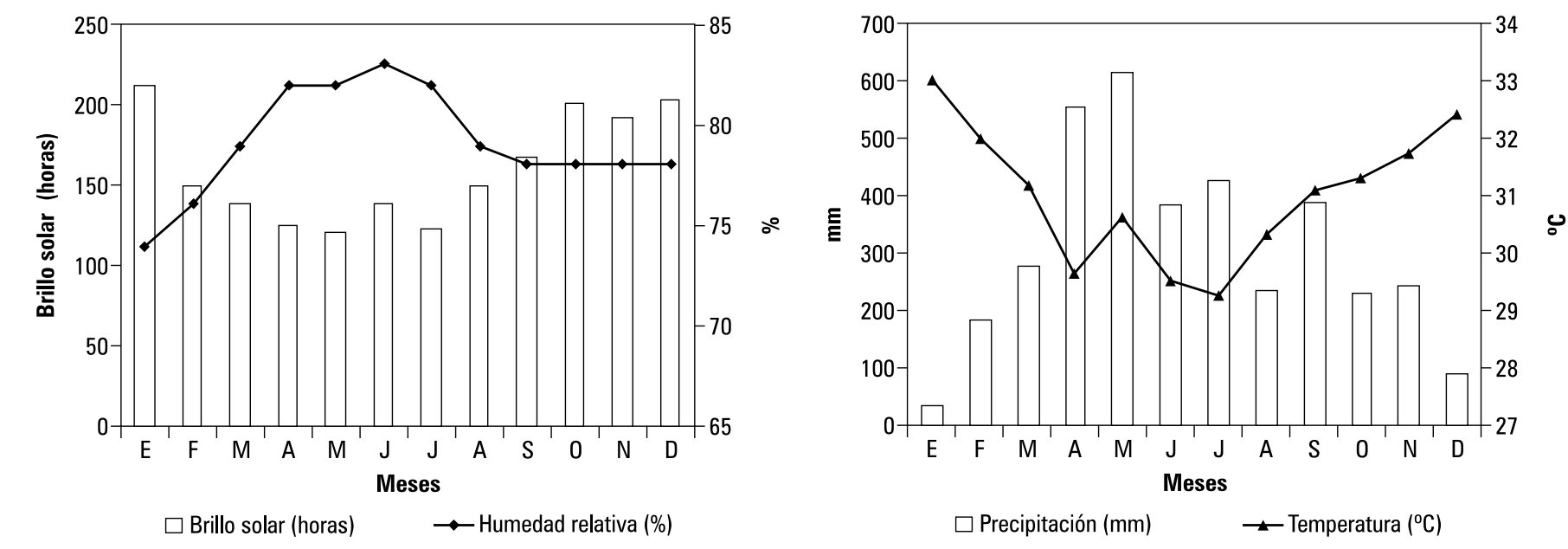

Figura 1. Información climatológica en la región de estudio. 


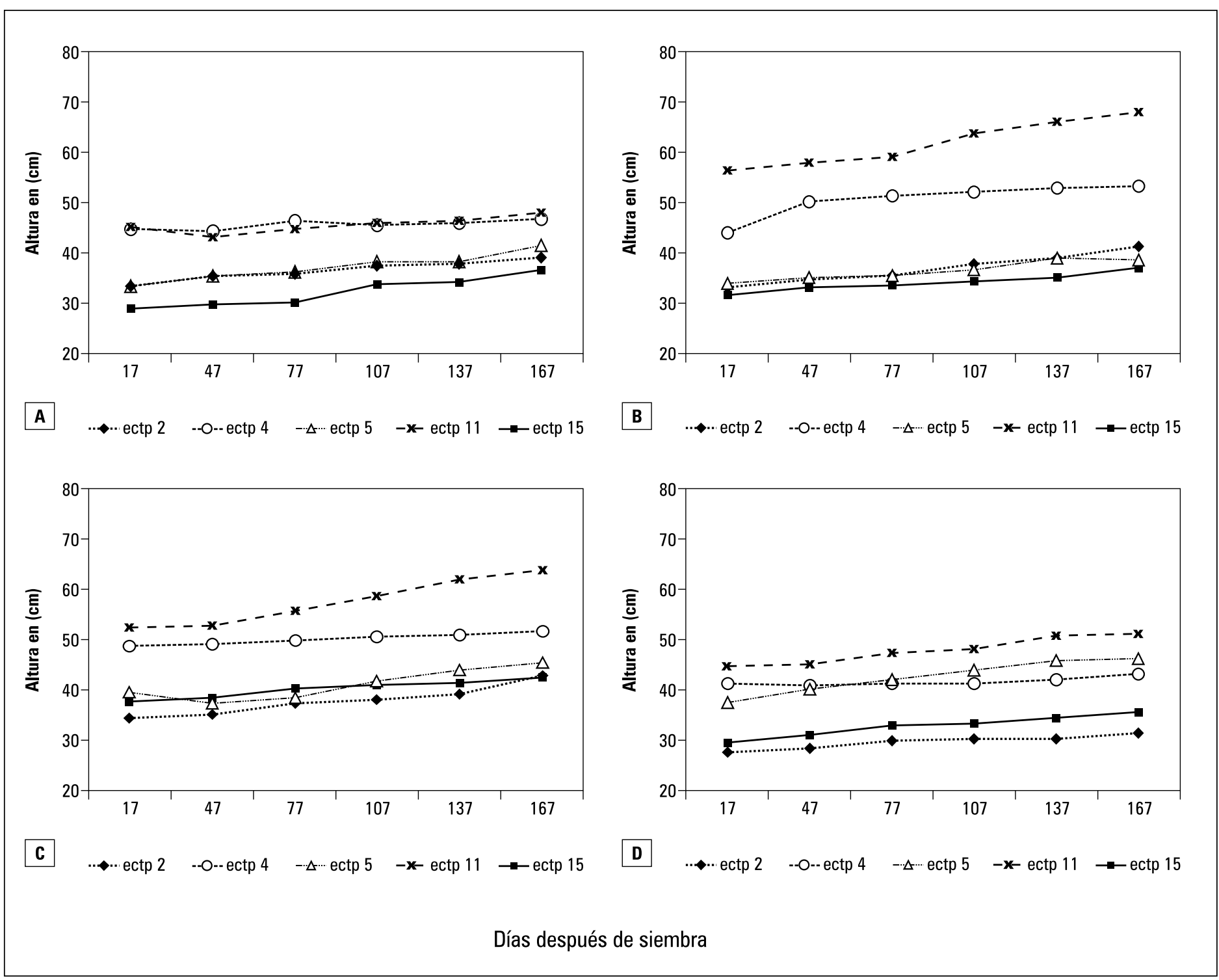

Figura 2. Crecimiento en altura en plantas de copoazú (Theobroma grandiflorum) en diferentes localidades. A: Bella Vista; B: Villa Nueva; C: Paraíso Amazónico; D: San Francisco 2.

to, que a su vez está regulada genéticamente, tal y como se pudo observar por la mejor respuesta de los ecotipos 3-004 y 3-011.

En cuanto al comportamiento entre localidades, se hallaron diferencias significativas $(P<0,01)$ en cuanto a la acumulación de materia seca se refiere. Estas diferencias son consecuentes con una mejor interceptación de radiación solar en las coberturas donde los árboles ganaron mejor altura. Según Salisbury y Ross (1992), la interceptación de radiación solar y su distribución en el perfil del dosel determinan la respuesta de la planta a la fotosíntesis. Dicho proceso es responsable de la producción de materia seca en un 90-95\%. De ahí se desprende la respuesta obtenida por los 5 ecotipos en las coberturas B y C, donde la acumulación de materia seca para todos los casos fue mayor que para las coberturas A y D. 

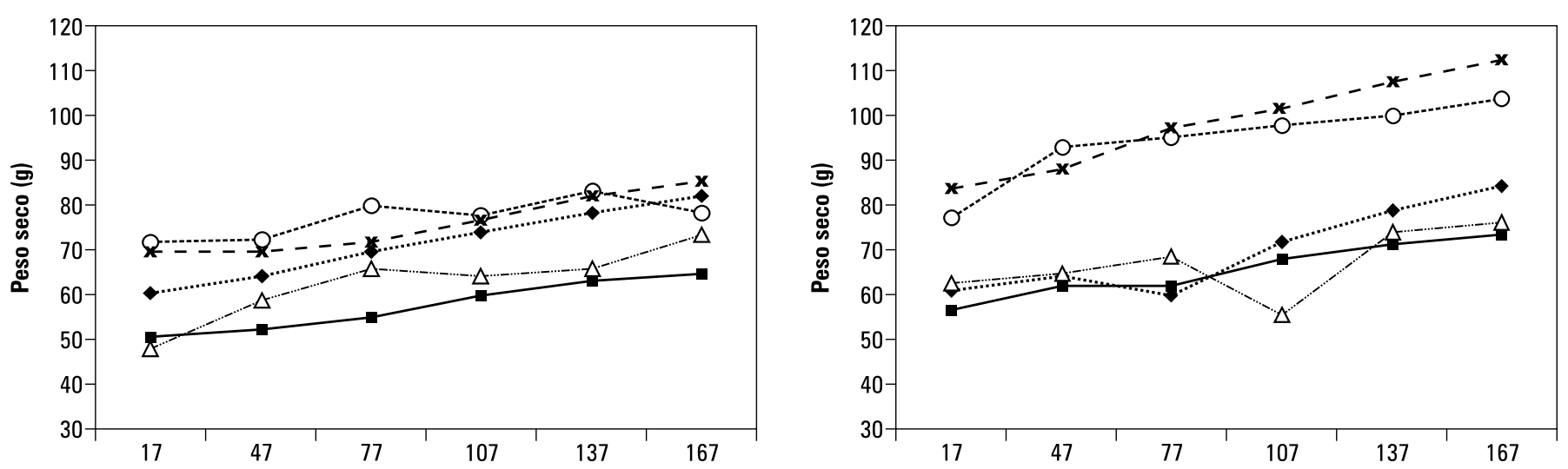

A

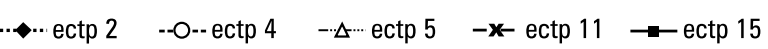
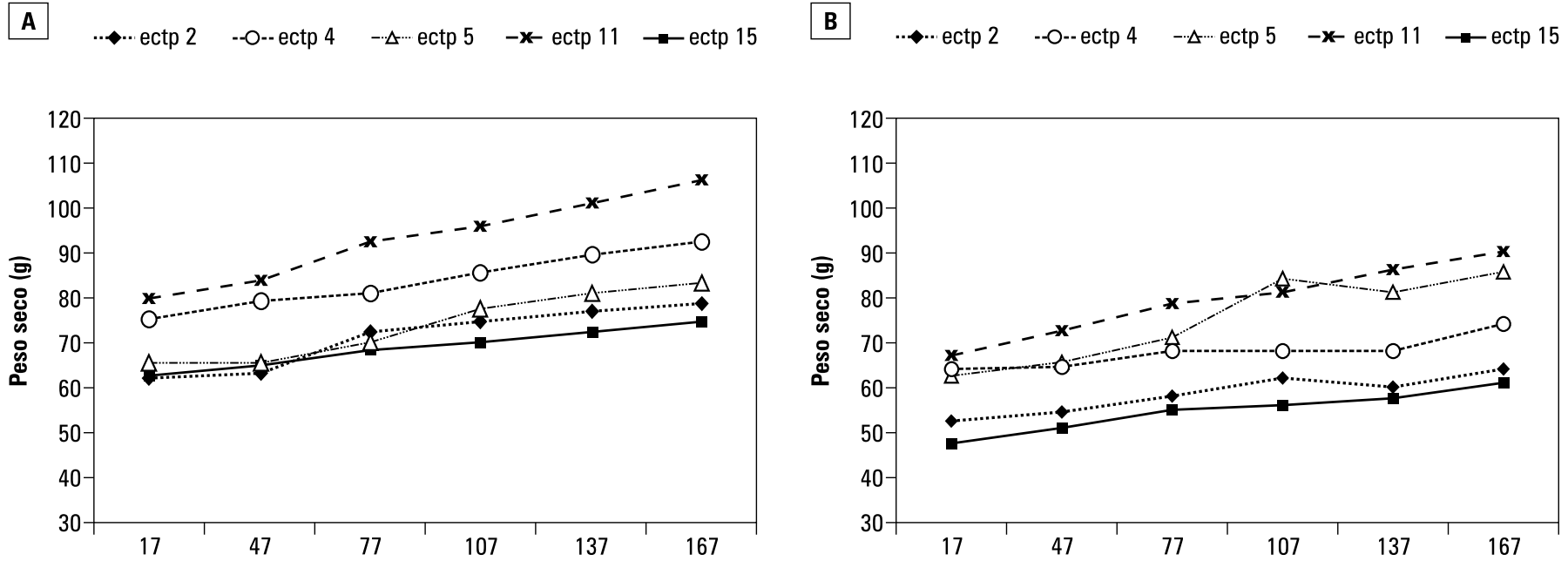

C

$\rightarrow-\cdots$ ectp $2 \quad--$-- ectp $4 \quad-\triangle-\cdots$ ectp $5 \quad$-x- ectp $11 \quad \rightarrow-\operatorname{ectp} 15$

D

ectp $2 \quad--$-- ectp $4 \quad-\Delta-\cdots \operatorname{ectp} 5 \quad-\mathbf{x}-\operatorname{ectp} 11 \quad \rightarrow-\operatorname{ectp} 15$

Días después de siembra

Figura 3. Acumulación de peso seco en plantas de copoazú (Theobroma grandiflorum) en diferentes localidades. A: Bella Vista; B: Villa Nueva; C: Paraíso Amazónico; D: San Francisco 2.

\section{Comportamiento del área foliar}

Se encontró que la expansión del área foliar en los cinco ecotipos se manifestó de manera fluctuante durante los 167 dds (figura 4). El aumento de la expansión foliar durante el periodo de evaluación no fue significativo, lo cual ratifica que en este periodo se dio una mayor tendencia a acumular materia seca en el tallo y de ahí la mayor expresión de crecimiento en esta variable. De otra parte, se encontró que el área foliar sí presento diferencias significativas para cada ecotipo, lo cual discrimina las posibilidades de una mayor interceptación de radiación solar. Este comportamiento se evidencia cuando en la cobertura de $15,8 \%$ y de manera análoga en la de $48,7 \%$, hubo un mayor aumento del área foliar como respuesta al sombreamiento, mostrando así la alta plasticidad de este órgano. Fernández (1982) y Jarma et al. (1999) reportan que la planta a bajos niveles de luz procura la expansión máxima de la superficie foliar para captar con mayor eficiencia la luz disponible. 

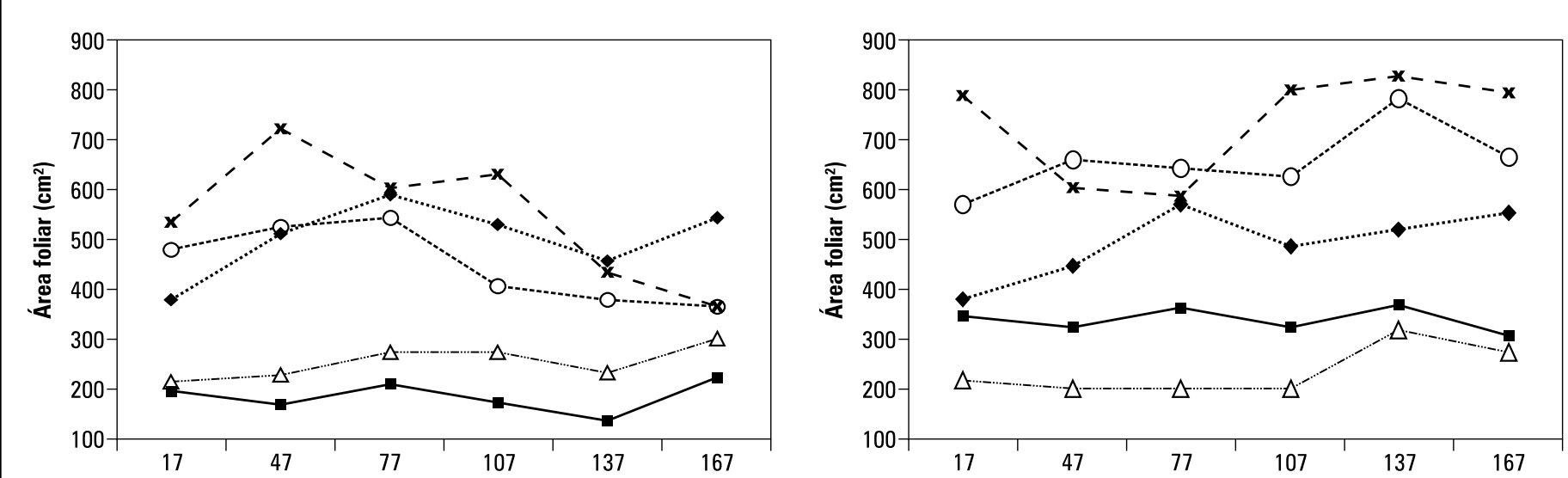

A
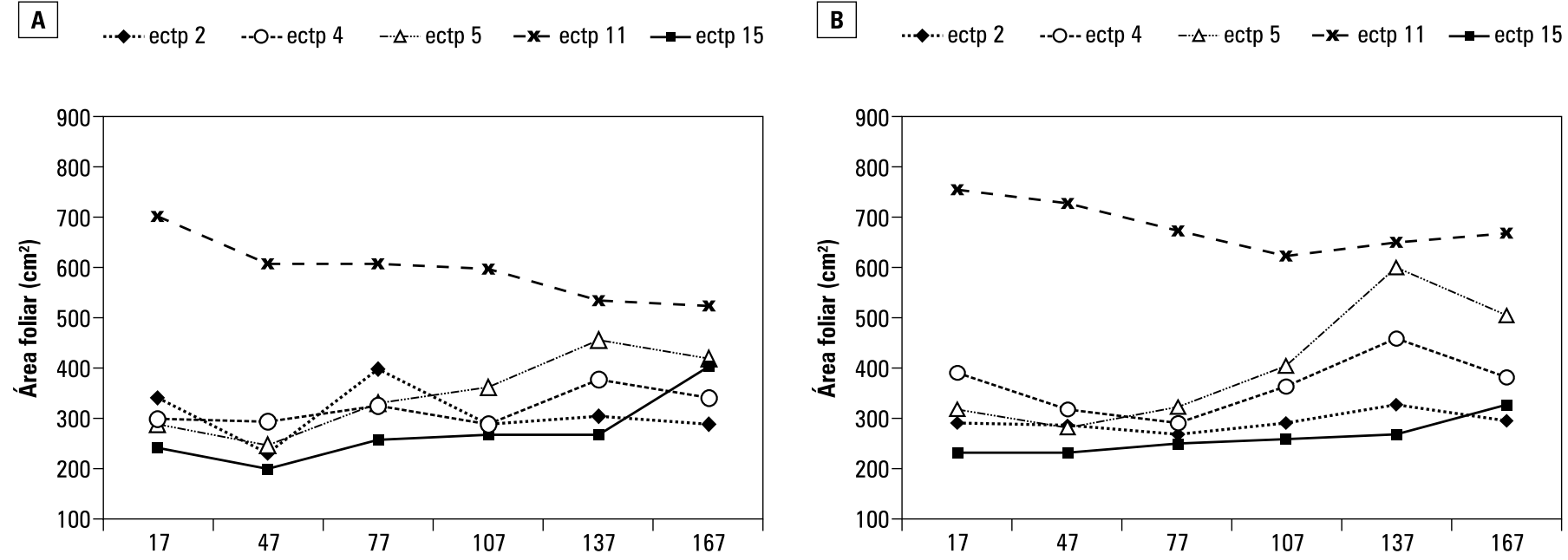

C

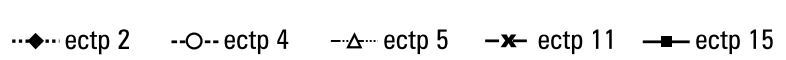

Días después de siembra

Figura 4. Área foliar en plantas de copoazú (Theobroma grandiflorum) en diferentes localidades. A: Bella Vista; B: Villa Nueva; C: Paraíso Amazónico; D: San Francisco 2.

Vale la pena resaltar que la mayor área foliar indistintamente de la localidad bajo la cual se desarrolló, la presentó el ecotipo 3-011 lo cual es una respuesta clara a la interacción ecotipo-ambiente. Para las plantas expuestas a competencia por luz se generan cambios en el área foliar y en el índice del área foliar, los cuales inducen una señal para los sistemas de control a nivel genético frente a condiciones de sombreamiento por plantas vecinas (Koller, 1990). Al parecer el co- poazú responde de manera plástica, aclimatándose a los diferentes niveles de RFA (Radiación Fotosintética Activa) respuestas que según Ballaré et al. (1995) involucran, tanto la morfología de la planta completa, como la fisiología de los cloroplastos.

Al observar las respuestas de los materiales en ganancia de peso seco y área foliar a medida que disminuye el grado de cobertura, se observó que 
el copoazú tiende a ser una especie facultativa de sombra, ya que exhibe características especiales de aclimatación al sol. Sin embargo, si la planta se somete después de un periodo de sombra a un periodo prolongado de alta radiación, es probable que las hojas sufran el fenómeno de fotoinhibición y se ocasione un daño considerable al aparato fotosintético reflejado en valores bajos de ganancia de materia seca y de área foliar en la cobertura de menor valor (TCA, 1999).

\section{COMPORTAMIENTO DE LOS ÍNDICES DE CRECIMIENTO}

El análisis de varianza sobre los índices de crecimiento, no presentó diferencias significativas en efectos principales y en sus interacciones. Gardner et al. (1984) argumentan que la relación entre la masa de la planta y el área foliar es lineal en los estados iniciales de crecimiento, mas no para las fases finales, donde el desarrollo del área foliar puede exceder los aumentos de masa seca y viceversa, de ahí puede relacionarse el comportamiento poco diferenciado obtenido en este estudio para índice como la TAN.

Para la TCR se observó que el incremento en peso seco por unidad de materia seca acumulada y por unidad de tiempo es variado entre los ecotipos, donde su tendencia es similar para las cuatro condiciones, coincidiendo con los resultados obtenidos en la TAN. Si comparamos las variaciones en el TCR y la TAN, se encuentra que el comportamiento es homogéneo. Por lo tanto, la conducta de los dos se puede explicar de forma análoga, pues son dependientes de la fotosíntesis, respiración, condiciones ambientales, tamaño de las hojas y estructura funcional de la planta (Gardner et al., 1984).

En el caso del IAF, al analizar el comportamiento de los ecotipos, se observó mayor área ocupada por las plantas que producción de tejido foliar, esto se debe a la mínima variación en el área foliar que presentaron los ecotipos durante el pe- riodo de evaluación. Por otro lado estuvo fuertemente afectado por las coberturas bajo las cuales se desarrollo el análisis y su interacción con el componente genético de cada material y ello se puede corroborar por los estados diferenciales entre doseles dado que el IAF expresa el área de hojas que convertirá la energía lumínica en energía química. Diversos autores han demostrado que mínimos valores de tasas de crecimiento relativo se observan después de valores máximos de IAF (Gómez et al., 1999). En este estudio se observó que los máximos valores de IAF se dieron en etapas tempranas de desarrollo (17 a $47 \mathrm{dds}$ ) lo cual se correlaciona con valores más bajos de TCR.

\section{RELACIÓN DEL ÁREA FOLIAR}

Se observó un efecto en la interacción genotipoambiente en relación con la intensidad de sombra que se proyecta en cada una de las plantas, las cuales influyen en el aumento de materia seca como la formación de nueva área foliar a través del tiempo (figura 5).

De acuerdo con Bjorkman (1981) las plantas pueden ajustarse a un ambiente de menor radiación, aumentando el área foliar especifica, lo cual se hace evidente en este caso cuando se observa una tendencia a incrementar la RAF en los cinco ecotipos bajo la influencia de la mayor cobertura (47,8\%).

De acuerdo con Gómez et al. (1999), la RAF es una medida del balance entre capacidad fotosintética potencial y el costo respiratorio potencial. En este caso fue variable significativamente entre los ecotipos estudiados y entre cada localidad, y no manifiesta una tendencia clara, lo cual indicaría que el balance entre la capacidad fotosintética y el costo respiratorio es inherente a cada material. En el caso del ecotipo 11, este índice demostró ser plástico en el sentido de aumentar cuando el nivel de sombreamiento fue menor (cobertura B 15,8\%), lo cual puede ser considerado como una respuesta en autocom- 


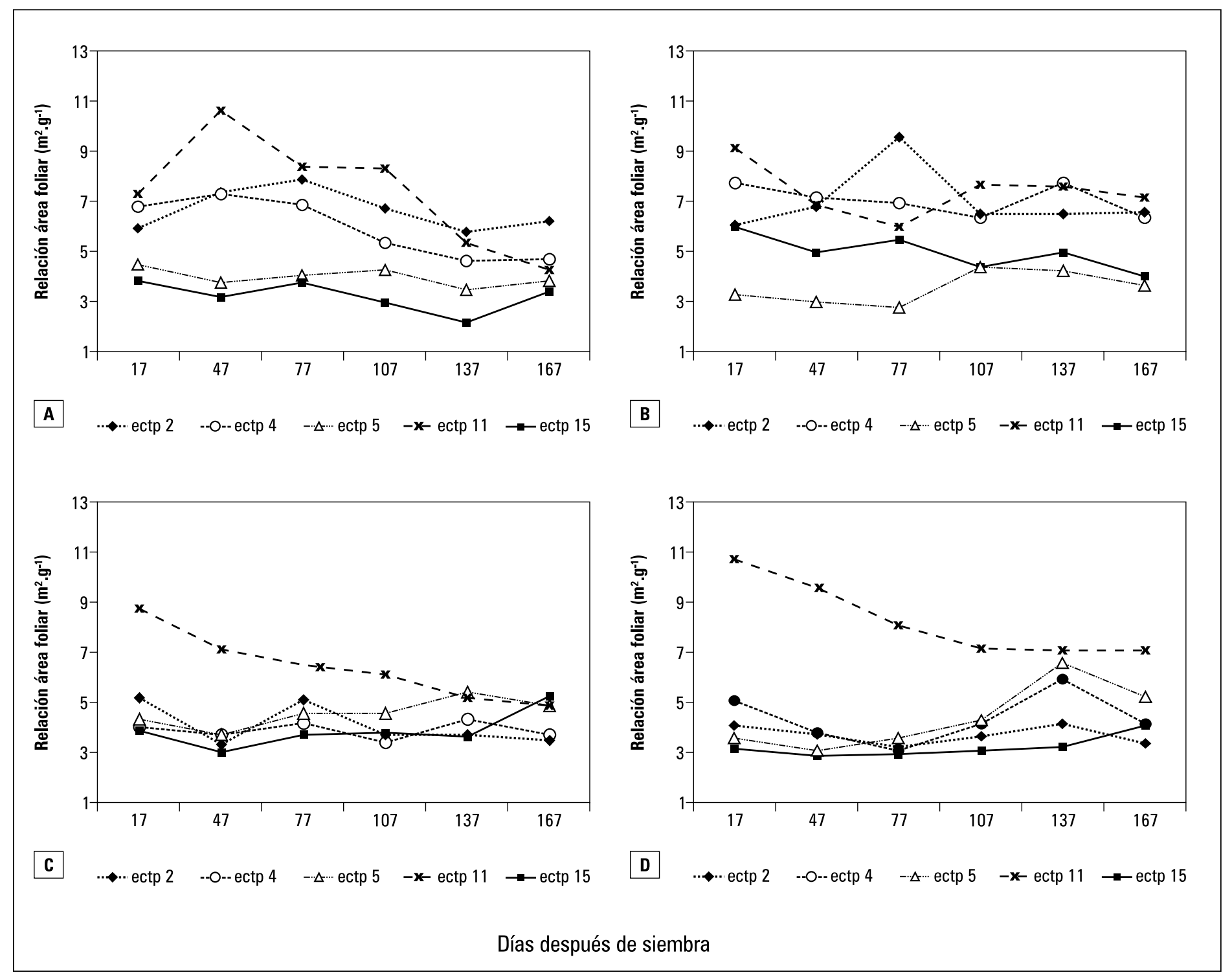

Figura 5. Relación de área foliar en plantas de copoazú (Theobroma grandiflorum) en diferentes localidades. A: Bella Vista; B: Villa Nueva; C: Paraíso Amazónico; D: San Francisco 2.

pensación de la planta para captar un máximo posible de energía solar disponible para la asimilación clorofílica en condiciones de baja luminosidad. (Jarma et al., 1999).

En todos los casos se evidencia que la RAF comienza a estabilizarse en distintos momentos del ciclo de evaluación, lo que indica la influencia que la cobertura ejerce sobre el momento de finalización del periodo de adaptación de los materiales asociado al componente genético de cada material.

\section{DURACIÓN DEL ÁREA FOLIAR}

La duración del área foliar (figura 6) describe la extensión y duración de las hojas como órgano interceptor de luz (Evans 1972; Hunt 1978). 

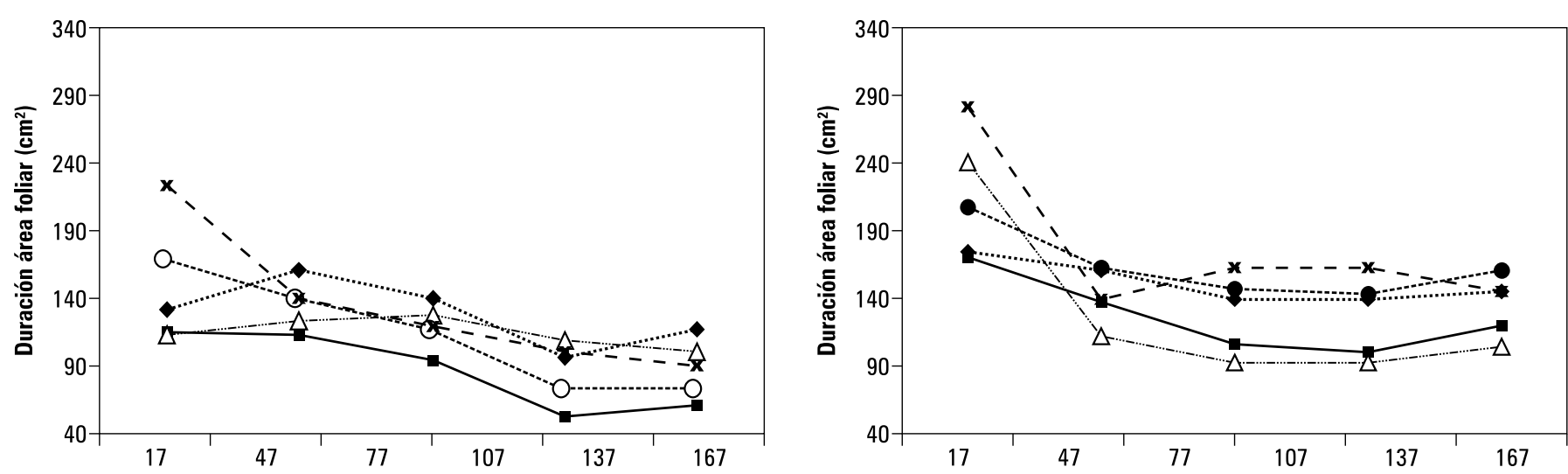

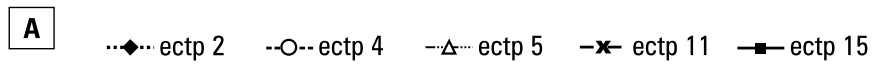
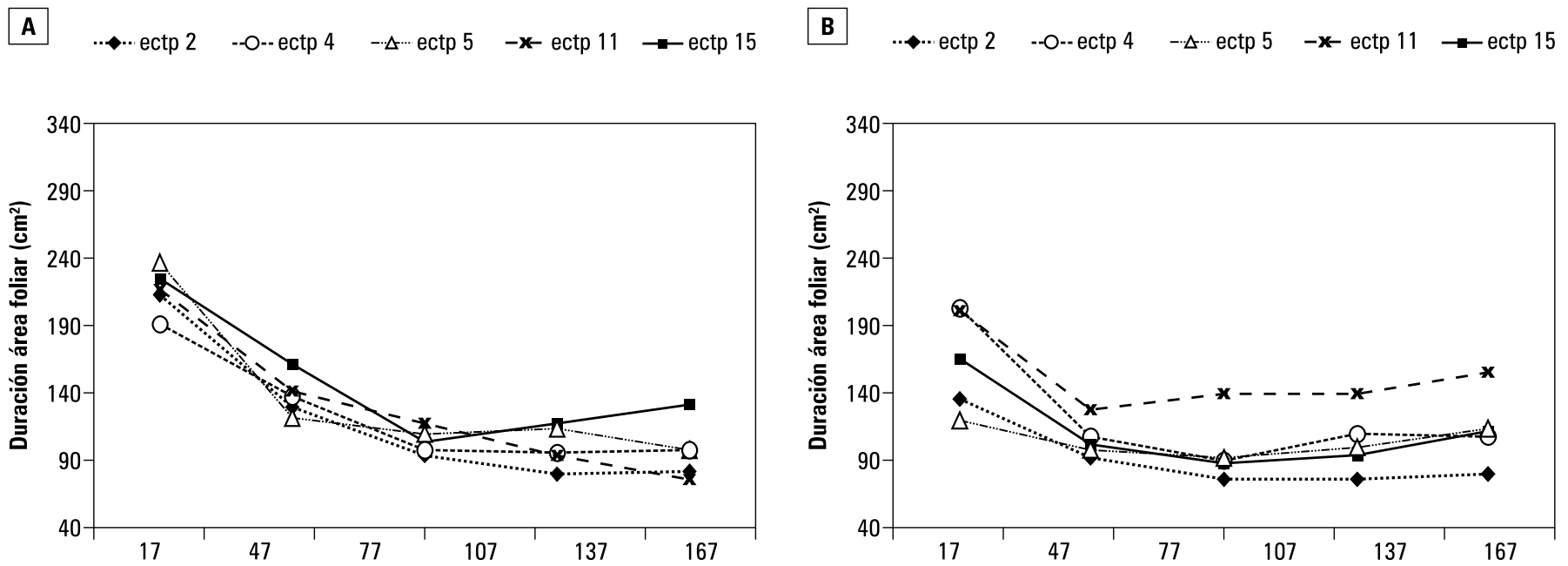

C

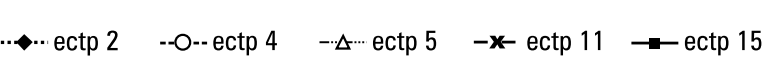

D

$\cdots \leftrightarrow$ ectp $2 \quad-$-O--ectp $4 \quad-\triangle-\cdots \operatorname{ectp} 5 \quad-\mathbf{x}-\operatorname{ectp} 11 \quad \rightarrow-\operatorname{ectp} 15$

Días después de siembra

Figura 6. Duración del área foliar en plantas de copoazú (Theobroma grandiflorum) en diferentes localidades. A: Bella Vista; B: Villa Nueva; C: Paraíso Amazónico; D: San Francisco 2.

Este índice es muy importante porque representa la duración del funcionamiento de la superficie asimiladora y sirve para interpretar el costo energético de la formación de la unidad de superficie foliar y su rendimiento en la producción de asimilados (Paez et al., 2000). En general, el DAF mostró tendencia al incremento en plantas desarrolladas a mayor cobertura o sombra, aunque la respuesta fue muy elástica, es decir, puede ajustarse a distintas circunstancias y en todos los ambientes. Los valores máximos de DAF se dieron hasta los primeros 47 días después de siembra y corresponden con periodos donde el área foliar fue de mayor magnitud y persistencia.

\section{ANÁLISIS DE REGRESIÓN LINEAL}

El análisis de regresión se desarrolló para los ecotipos 3-004 y 3-011, que resultaron sobre- 
salientes con respecto a los otros materiales en términos de altura y materia seca. Se seleccionaron para este análisis las variables fisiológicas de mayor respuesta indistintamente de la cobertura bajo la cual se obtuvieron, con el objeto de aproximar al comportamiento ecofisiológico de estos materiales.

Se generaron modelos polinomiales con y sin intercepto para las variables área foliar, peso seco, duración de área foliar y relación de área foliar, encontrándose mejor ajuste en los modelos polinomiales de tercer orden sin intercepto.

Los coeficientes de regresión, que determinan el ajuste del modelo, oscilaron entre el 79 y el 95\% y se dio significancia estadística en todos los términos del modelo. Con estas ecuaciones se grafica la tendencia respecto al tiempo de cada variable hasta los $257 \mathrm{dds}$, momento en el cual se considera habría un $100 \%$ de formación tricotómica que afecta las relaciones fisiológicas. El padrón tricotómico se caracteriza, inicialmente, por el crecimiento vertical del eje principal, el cual originará el tronco, que al alcanzar una altura de 40 a $50 \mathrm{~cm}$, emite en su porción terminal tres ramificaciones laterales de crecimiento plagiotrópico. En seguida, se desarrolla una nueva yema en el centro de las ramificaciones iniciando un nuevo ciclo de crecimiento del eje principal de la planta que al llegar a una altura de 70 a 100 $\mathrm{cm}$, forma otro conjunto de ramificaciones plagiotrópicas en las extremidades. Estos crecimientos periódicos y ordenados, caracterizados por el desarrollo de una yema de crecimiento ortotrópico y tres de crecimiento plagiotrópico, ocurren hasta que la planta llega a la madurez (TCA, 1999).

Es de esperarse que para las cuatro variables analizadas las tendencias sean estables hasta los 167 dds, coincidente con el periodo de lento crecimiento (Lyons y Sánchez, 1983), momento a partir del cual se inicia la fase logarítmica de crecimiento, la cual se da a partir de los 197 dds. Se considera que una vez se da la diferenciación tricotómica, la dinámica cambia por la emisión de las ramificaciones plagiotrópicas que afecta la dinámica fotosintética y la ganancia de materia seca. De acuerdo con estas tendencias, el ecotipo 11 puede presentar el mejor comportamiento bajo condiciones similares a las que se evaluaron en esté estudio.

\section{CONCLUSIONES}

- Los resultados obtenidos en el AF, las TAN, TCR, la RAF y el IAF presentaron diferencias significativas en cada uno de los ecotipos, expresando de esta forma una importante interacción genotipo/ambiente, donde se observa cierta plasticidad por parte de los materiales en su respuesta a cada una de las coberturas.

- El análisis de crecimiento indicó que los ecotipos 4 y 11 presentaron un comportamiento fisiológico similar y sobresaliente respecto a los demás ecotipos durante la fase vegetativa temprana, especialmente en parámetros como materia seca, altura, área foliar y DAF, los cuales son determinantes fisiológicos para el crecimiento y desarrollo de las plantas.

- Fue evidente que la interacción genotipo/ambiente se expresó en la capacidad que tiene cada una de las plantas para adaptarse a un ambiente determinado diferenciando el crecimiento bajo diversas condiciones. Para este caso los ecotipos 4 y 11 presentaron mejor desenvolvimiento en la cobertura ralas $(15,8 \%)$ demostrando una buena adaptabilidad en este tipo de ambientes.

- Los ecotipos 3-004 y 3-011 se seleccionan además por presentar características morfoagronómicas ideales ya, que tienen una de las producciones más altas registradas para la colección, pertenecen a diferentes grupos moleculares reflejando una mayor diversidad en el sistema y expresan diferentes respuestas a la tolerancia a las principales enfermedades que atacan el cultivo de copoazú en la región. 


\section{REFERENCIAS BIBLIOGRÁFICAS}

Ballaré, C.L.; P.W. Barnes y S.D. Flint. 1995. Inhibition of hypocotyl elongation by ultraviolet-B radiation in de-etiolating tomato seedlings: I. The photoreceptor. Physiol. Plant. 93, 584-592.

Bjorkman, O. 1981. Responses to different quantum flux densities. En: Encyclopedia of plant physiology: Plant ecology I. Springer-Verlag, New York. pp. 57-107.

Donoso, C. 1981. Ecología forestal, el bosque y su medio ambiente. Facultad de Ciencias Forestales, Universidad Austral de Chile, Valdivia. 369 p.

Early, E.B. 1996. Effects of shade on maize production under field conditions. Crop Sci. 6, 1-7.

Lopes, N.F.; M.A. Oliva; J.G. Freitas; E. Melges y N.E. de M. Beltrão. 1982. Análise de crescimento e conversão da energia solar em feijoeiro (Phaseolus vulgaris L.) submetido a três níveis de densidade do fluxo radiante. Ceres Viçosa 29, 586-606.

Evans, G.C. 1972. The quantitative analysis of plant growth. Studies in ecology. Vol 1. Blackwell Scientific Publication, London. pp. 45-68.

Gardner, F.; R. Pearce y R. Mitchell.1985 Physiology of crop plants. Iowa State University Press, Aimes. pp. 187-208.

Gómez, C.; C. Buitrago; M. Cante y B. Huertas. 1999. Ecofisiología de la papa (Solanum tuberosum). Revista Comalfi 26, 42-55.

Hunt, R. 1978. Plant growth análisis. Studies in biology 96. Edward Arnold Publishers, London. 67 p.

Hunt, R.; J. Warren Wilson; D.W. Handy y D.G. Sweeney. 1984. Integrated analysis of growth and light interception in winter lettuce. I. Analytical methods and environmental influences. Ann. Bot. 54, 743-757.
Jarma, A.; C. Buitrago y S. Gutiérrez, S. 1999. Respuesta de crecimiento de la habichuela (Phaseolus vulgaris L. Var. Blue Lake) a tres niveles de radiación incidente. Revista Comalfí 26, 62-73.

Koller, D. 1990. Light drive leaf movements. Plant Cell Environ., $615-632$.

Little T.M. y F.J. Hills. 1976. Métodos estadísticos para la investigación en la agricultura. Editorial Trillas, Mexico. pp. 59-65.

Lynois. E. y J. Sanchez. 1983. Análisis de crecimiento de la fase vegetativa y de formación de espiga de tres cultivares de cebada (Hordeum vulgare) bajo tres condiciones de siembra. Trabajo de grado. Facultad de Agronomía, Universidad NacionaldeColombia.129p.

Magalhaes, A.C. y J.C. Motojo. 1971. Effect of solar radiation on the growth parameters and yield of two varieties of common bean (Phaseolus vulgaris). Turrialba 21, 165-168.

Paez. A.; V. Paz y J. López. 2000. Crecimiento y respuesta fisiológica de plantas de tomate cv. Río Grande en la época mayo-julio. Efecto de sombreado. Rev. Fac. Agron. (LUZ) 17, 173-184.

Rangel, O.; P. Lowy y M. Aguilar. 1997. Colombia diversidad biótica II. Tipos de vegetación en Colombia. Editorial Guadalupe Ltda., Bogotá. pp. 59-87.

Rojas, M. 1993. Fisiología vegetal aplicada. Cuarta edición. Interamericana McGraw Hill.

Rojas, S.; J. Zapata; E. Varón y A. Pereira. 1996. Copoazú. Corpoica Macagual Florencia, Caquetá.

Salisbury, F. y C. Ross. 1994. Fisiología vegetal. Grupo Editorial Iberoamérica, Mexico. pp. 376-377.

Tratado de Cooperación Amazónica. 1999. Copoazú Theobroma grandiflorum (Willd ex Spreng) Schum. cultivo y utilización. Manual técnico. 